\title{
Comment la recherche concertée contribue à l'écologie territoriale
}

\author{
Jérôme Queste ${ }^{1,2, *}$ et Tom Wassenaar ${ }^{3,4}$ \\ ${ }^{1}$ CIRAD, UPR GREEN, 101, Antananarivo, Madagascar \\ 2 GREEN, Univ Montpellier, CIRAD, Montpellier, France \\ ${ }^{3}$ CIRAD, UPR Recyclage et Risque, 34398 Montpellier, France \\ ${ }^{4}$ Recyclage et Risque, Univ Montpellier, CIRAD, Montpellier, France
}

\begin{abstract}
Résumé - La concertation semble en passe de devenir une norme en matière de gestion des ressources naturelles et de l'environnement. Pourtant, son impact reste difficile à caractériser, ce qui génère une forme de désenchantement auprès de ses promoteurs. Au cours d'un projet de recherche concertée à La Réunion, nous avons cherché à mieux comprendre comment la concertation influence l'écologie territoriale. Pour ce faire, nous avons réalisé un double suivi, du processus de concertation et de ses produits d'une part, des changements en lien avec l'objet de la concertation survenant sur le territoire d'autre part. Le second suivi a conduit à une série de récits d'innovation au sein desquels nous avons pu mettre en évidence les contributions des produits de la concertation. Les produits de la concertation ayant contribué aux changements sont des apprentissages individuels et collectifs et une reproblématisation partagée de la situation. Cette reproblématisation que nous qualifions de mythe rationalisé se compose d'un problème à résoudre - le gaspillage de nutriments dû à une mauvaise gestion des déchets organiques - et d'une solution sociotechnique susceptible de résoudre ce problème - une filière intégrée de recyclage de ces déchets sous la forme de produits fertilisants normés. Ces produits contribuent aux changements en d'autres lieux que les arènes de concertation et selon d'autres agendas que celui du projet. Ils participent à un ensemble de microchangements non planifiés mais alignés dans une direction commune. Le mythe rationalisé assure ici une fonction de coordination informelle. Nos résultats suggèrent que la prise en compte du contexte dans lequel les processus participatifs se déroulent améliore l'évaluation des effets de la concertation. Ils soulignent également la pertinence du recours à une recherche concertée dans le domaine de l'écologie territoriale.
\end{abstract}

Mots clés : concertation / évaluation de l'impact / écologie territoriale / engrais organique / apprentissage

\begin{abstract}
How dialogue research contributes to territorial ecology. Dialogue is becoming a prerequisite for natural resource management. However, its impact assessment remains a problem, leading to a growing disillusion. We tried to better understand how the outputs of a dialogue-based research project could influence a territory's ecology. To do so, we monitored distinctively the dialogue process and its outputs on one hand, the changes occurring on the territory on the other hand. The later monitoring led to narratives highlighting the contribution of dialogue outputs. Relevant outputs are individual and collective learnings and a rephrasing of what we call a rationalized myth composed of an environmental problem - a waste of nutrients due to a poor recycling management - and a sociotechnical solution, an integrated recycling value-chain producing standardized fertilizers. These outputs are used as resources in other locations than the participatory workshops, following other agendas than the dialogue process. They contribute to a broad diversity of micro-changes and individual decisions, unplanned but aligned in a common direction. These results suggest to better consider dialogue processes as embedded in a territory. They also demonstrate the relevance of dialogue-research for territorial ecology.
\end{abstract}

Keywords: dialogue / impact assessment / territorial ecology / organic fertilizer / learning

\footnotetext{
$\overline{\text { *Auteur de correspondance }}:$ jerome.queste@cirad.fr
} 


\section{Le défi de l'évaluation des effets de la recherche concertée}

La complexité et l'importance croissante des problèmes de gestion des ressources naturelles renouvelables et de l'environnement ont conduit chercheurs et gestionnaires à expérimenter de nouvelles modalités de mise en œuvre de l'action publique (Le Bourhis, 2012). La participation se voit institutionnalisée par la déclaration de Rio en 1992, la convention d'Aarhus en 1998 et en France, plus récemment, par l'article 7 du fameux «Grenelle de l'environnement» de 2005. Elle s'affirme donc comme une norme de l'action publique environnementale (Barbier et Larrue, 2011). La relation entre participation et environnement est profonde. Les sciences de l'environnement ont davantage recours à des dispositifs participatifs que n'importe quel autre champ de recherche (Fazey et al., 2014). La participation des parties prenantes à l'action collective est à la base des concepts de cogestion de biens communs (Ostrom, 1990) comme les territoires, l'environnement ou les ressources naturelles renouvelables (Berkes, 2009; Olsson et al., 2004).

Le terme de participation recouvre une variété de pratiques. Plusieurs typologies et critères de dissociation sont proposés, notamment au sein de la littérature anglo-saxonne (Barbier et Larrue, 2011) qui rendent nécessaire une précision de notre objet d'étude. Par concertation, nous entendons «un processus de construction collective de questions, de visions, d'objectifs et de projets communs relatifs à un objet » (Beuret et al., 2006). Nous qualifions de recherche concertée une activité de recherche ayant recours à un processus de concertation. Une telle recherche constitue une forme particulière de recherche participative associant des représentants des parties prenantes à la construction des objets de recherche et à l'analyse des résultats.

Ce faisant, nous adoptons une vision substantielle de la participation. La recherche concertée se justifie d'abord pour améliorer la qualité de la gestion de l'environnement, enjeu que nous plaçons avant le renouvellement démocratique et l'amélioration des relations entre institutions et citoyens. Elle a pour objectif premier la production de connaissances scientifiques et ne s'inscrit donc qu'indirectement dans l'action publique.

L'engouement pour les dispositifs participatifs et leur institutionnalisation contraste avec le faible nombre et la dispersion des travaux de recherche visant à évaluer leurs effets sur l'action publique. La convergence de ces travaux est rendue délicate par la diversité des domaines couverts, des formes de participation et des procédures suivies (Mazeaud et al., 2012). Un premier ensemble de travaux s'est efforcé d'isoler les effets propres de la procédure participative ou des outils mobilisés comme la modélisation d'accompagnement (Étienne, 2011), les scénarios prospectifs (Biggs et al., 2007) ou les modèles mentaux (Lynam et al., 2007) sur la décision publique. Mazeaud et al. (2012) regroupent les effets identifiés par ces travaux en trois modalités que sont:

- une influence directe sur le contenu de politiques publiques;

- une influence indirecte sur les idées et les controverses ;

- une influence indirecte via l'apprentissage des acteurs.
Ces travaux s'appuient cependant sur un modèle «balistique» de l'action publique. Ce modèle place au centre de l'action publique un décideur unique prenant à un instant $t$ des décisions irrévocables, pour atteindre des objectifs explicites. Ce modèle est contesté par les récents travaux de sociologie de l'action publique qui insistent sur le caractère bricolé, voire aléatoire de la fabrique de l'action publique et sur l'importance des arrangements pratiques liés à la mise en œuvre de décisions floues (Lascoumes et Le Galès, 2007).

Un second ensemble de travaux considère que l'action publique consiste surtout à arbitrer une action collective complexe et polycentrique au sein de laquelle la décision n'est pas un point de passage obligé. Ces travaux prennent de la distance vis-à-vis des procédures pour aborder la concertation comme un processus encastré dans le système d'action sur lequel il prétend agir (Barbier et Larrue, 2011). La difficulté méthodologique de cette approche réside dans le fait qu'elle n'isole pas les arènes de concertation du reste de l'action collective, ce qui permet de mieux appréhender la manière dont se déroule cette action collective mais rend plus difficile la mise en évidence de liens de causalité.

Les recherches présentées dans cet article s'inscrivent dans cette seconde perspective. Elles visent à mettre en évidence les effets d'un projet de recherche concertée non pas sur les participants mais sur le système d'action qui fait l'objet de cette concertation, c'est-à-dire sur les pratiques, routines et actions concrètes du quotidien des acteurs d'un territoire. $\mathrm{Ce}$ faisant, nous formulons l'hypothèse selon laquelle un tel projet de recherche contribue à l'action publique en dépit de l'absence de mandatement formel.

\section{Cas d'étude: Le projet de recherche concertée Girovar et l'écologie territoriale}

L'étude porte sur le projet de recherche Girovar (Gestion intégrée des résidus organiques par leur valorisation agronomique à La Réunion) conduit et facilité par les deux auteurs de 2011 à 2014 à La Réunion. Ce projet combinait concertation multi-acteurs et multi-niveaux et recherche opérationnelle. L'île de La Réunion était en 2011 confrontée à un problème de gestion de matières organiques : on y observait simultanément une augmentation des volumes de résidus organiques produits (effluents d'élevage, déchets verts et des boues d'épuration notamment), une diminution des surfaces agricoles disponibles et une augmentation des charges d'importation d'engrais chimiques de synthèse.

Le point de départ du processus de concertation fut une proposition technique portée par le projet consistant à organiser une filière territoriale de recyclage des résidus organiques à l'échelle d'une communauté d'agglomération située dans l'Ouest de La Réunion. Cette proposition correspond à une stratégie d'Écologie industrielle et territoriale définie comme la mise en commun volontaire de ressources par des acteurs économiques d'un territoire, en vue de les économiser ou d'en améliorer la productivité (CGDD, 2014). Pour les chercheurs, les flux de matière organique sont des objets de recherche interdisciplinaires sur lesquels travaillent chimistes, agronomes, pédologues, aménageurs et ingénieurs. En termes d'action publique, une telle filière apporte des réponses à plusieurs enjeux territoriaux brûlants 
mais nécessite de mettre en relation directe des secteurs d'activités distincts comme la collecte et le traitement de déchets, l'élevage, le traitement des eaux usées, le maraîchage et l'industrie sucrière.

La concertation fut pilotée par une petite équipe projet constituée d'une dizaine d'ingénieurs, de chercheurs et de cadres techniques d'administrations, dont les auteurs de cet article. Elle bénéficia d'un appui institutionnel de la part de la préfecture, de la communauté d'agglomération «Territoires de la Côte Ouest » (TCO) et d'un élan lié à la montée en puissance des réglementations environnementales européennes. Le dispositif de concertation fut articulé autour de trois arènes intersectorielles fonctionnant selon des modalités distinctes et associant :

- des personnes directement impliquées dans la manipulation des matières organiques (éleveurs, agents, agriculteurs) invitées pour participer à des ateliers de coconstruction d'un premier jet de scénarios possibles, puis à des ateliers d'évaluation de la pertinence de ces scénarios retouchés par les deux autres arènes;

- des personnes possédant une expertise technique cooptées pour participer à des réunions de travail portant sur les aspects techniques de ces scénarios ;

- des représentants institutionnels des différents secteurs invités à un comité de pilotage pour prononcer un avis institutionnel sur chaque scénario.

Entre chacun de ces évènements, l'équipe projet synthétisait les conclusions et conduisait des études de faisabilité technique, environnementale et économique des scénarios proposés. Les résultats de ces études étaient ensuite à nouveau restitués aux trois arènes de manière séquentielle (Queste, 2016).

Durant les trois années du projet, plusieurs controverses portant sur la disponibilité de surfaces d'épandage possible, sur l'usage des boues d'épuration en agriculture, sur la volonté des éleveurs de volaille et des raffineries de sucre à adhérer à une filière commune et sur le partage des bénéfices de la filière furent ouvertes puis reformulées ou refermées (Queste, 2016). Ce projet aboutit à la production et à l'évaluation de deux scénarios de mise en place d'une filière de production et de distribution de fertilisants organiques normés (Wassenaar et al., 2015). Le premier scénario prévoit l'installation d'une nouvelle unité de traitement qui effectuerait le mélange et le co-compostage d'une combinaison de litières de volailles, de lisier de porcs et de déchets verts broyés pour produire un fertilisant commercialisable tel quel. Le second scénario ajoute une deuxième unité de traitement qui produirait des fertilisants plus concentrés en mélangeant le premier fertilisant à d'autres résidus comme la vinasse de distillerie, les fientes de poules pondeuses et des compléments chimiques. L'évaluation des scénarios conclut à leur faisabilité technique et agronomique, à son innocuité environnementale mais à un équilibre économique précaire. Elle souligne également l'absence de références scientifiques sur l'efficience des fertilisants organiques, peu connue en général et dépourvue de références locales.

La concertation ne conduit pas ici à une décision collective de mettre en œuvre l'un ou l'autre des scénarios. Pour autant, l'évaluation de ce projet révèle des changements significatifs sur la manière dont circulent les matières organiques sur ce territoire.

\section{Mettre en évidence les liens entre produits de la concertation et changements}

Pour structurer notre étude, nous nous sommes inspirés du cadre d'évaluation du chemin de l'impact, proposé par Douthwaite et al. (2003) et adopté par plusieurs organismes de recherche en agronomie (Barret et al., 2017; Joly et al., 2015). Ce cadre propose une modélisation des fils d'action menant des activités du projet à la réalisation d'impacts durables. Il distingue les produits des activités (outputs) du projet (documents, infrastructures, connaissances, apprentissages et idées) des changements (outcomes) observés sur le terrain au niveau micro ou méso (nouvelle organisation, changement de pratiques, renforcement du capital social). Ces changements sont des étapes intermédiaires pouvant le cas échéant conduire à des impacts définis comme une évolution de variables au niveau macro comme le revenu des agriculteurs, le niveau de pollution d'une nappe phréatique ou le taux de déforestation annuel. Une présentation synthétique de ces résultats, obtenus en appliquant la méthode Impress (Barret et al., 2017) est proposée dans la figure 1.

Toutefois, l'identification de liens de causalité entre les produits et les changements constatés, de part et d'autre de l'«attribution gap» (Kuby, 1999 cité par Douthwaite et al., 2003) demeure une zone d'ombre de la méthode.

Pour identifier ces liens de causalité, nous avons mis en place un double dispositif de suivi dès les premières étapes de conception du projet. D'un côté, nous avons collecté tout au long du projet les traces permettant de rendre compte de l'apparition de produits du projet, intentionnels ou non, comme l'évolution du périmètre social constitué par les participants aux ateliers, l'émergence et la résolution de controverses, la co-construction de connaissances nouvelles.

De l'autre côté, des études ethnographiques ont été conduites en début et en fin de projet pour rendre compte des changements survenus au sein de deux circuits de valorisation des déchets organiques susceptibles d'être affectés par le projet : l'utilisation de litières de volaille en maraîchage et la production et la distribution de compost de déchets verts. $\mathrm{La}$ grille d'analyse utilisée a été conçue spécifiquement pour cette étude. Parce que l'objet central de la concertation était la circulation des matières organiques entre différents acteurs, la grille d'analyse fut centrée sur les situations d'action concrètes au cours desquelles ces matières changent de main, les transactions et sur les dispositifs institutionnels de cadrage de ces transactions (Dufy et Weber, 2007), qui sont : le nettoyage des bâtiments d'élevage de volaille (130 questionnaires, 2 observations) et le ballet des camions de collecte de déchets verts autour de la station de compostage (24 entretiens, 7 jours d'observation). D'autres informations concernant ces changements ont été obtenues aux cours des ateliers de concertation. Enfin, à l'issue du projet, un atelier d'évaluation et une série de 27 entretiens semi-directifs conduits auprès des principaux partenaires du projet ont également produit de nouvelles données. Un exercice de triangulation de ces quatre sources de données a été réalisé pour en renforcer la validité et l'épaisseur.

Dans un second temps, nous avons reconstitué l'histoire de chacun des changements identifiés sous la forme de récits. Cette reconstitution a posteriori s'est appuyée sur la 


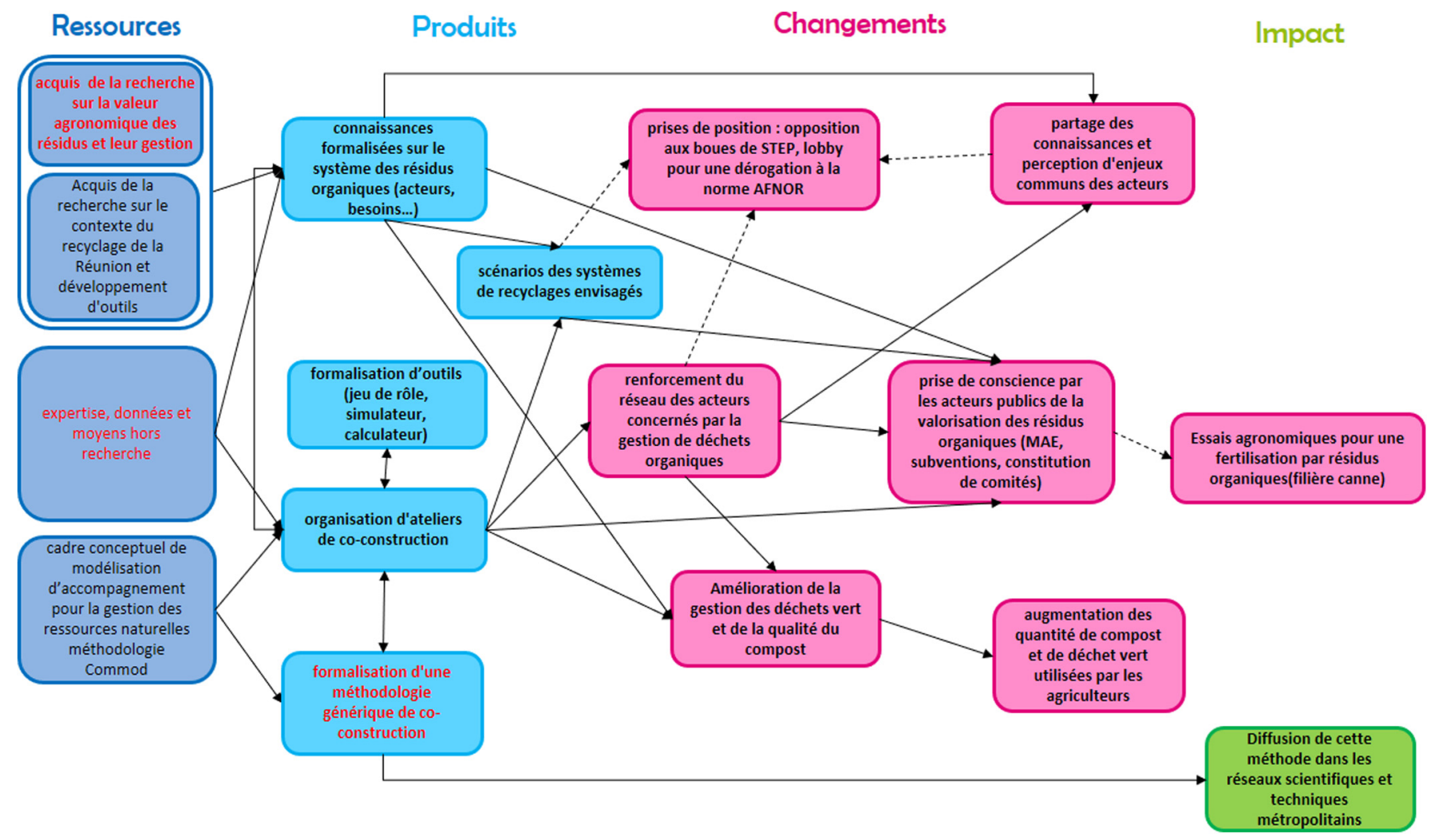

Fig. 1. Chemin de l'impact du projet Girovar à la Réunion selon l'équipe projet.

Fig. 1. Impact pathway of the Girovar project according to the project team.

confrontation d'entretiens semi-directifs, de traces écrites et matérielles et d'observations directes. L'analyse de ces récits a mis en évidence les contributions des produits du projet aux changements.

\section{Des contributions diverses à de nombreux micro-changements}

En 2015, le processus de concertation du projet Girovar avait contribué à la réalisation des changements présentés ci-dessous. Les personnes interrogées n'ont cependant pas attribué directement ces changements à la concertation proprement dite. Cette dernière est perçue comme un instrument d'influence et d'accompagnement de dynamiques déclenchées par ailleurs.

Un premier changement est la sortie de crise de l'activité de traitement des déchets verts de la communauté d'agglomération TCO. En 2010, cette activité s'appuie sur deux stations de traitement saturées et sujettes à des départs d'incendies récurrents. Le TCO envisage d'abandonner le compostage des déchets verts au profit d'une filière d'incinération. En 2014, ces deux stations, de compostage du Port et de broyage de Saint-Leu, fonctionnent à plein régime et peinent à satisfaire la demande en compost de déchets verts. La contribution du projet Girovar est identifiée à plusieurs niveaux : la production de connaissances par les agents du TCO, une meilleure compréhension des attentes de leurs clients potentiels et une «prise de conscience de la valeur du compost de déchets verts» accompagnent un processus d'apprentissage organisationnel au sein du TCO qui aboutit à une refonte du système de contrôle qualité de l'ensemble du circuit, depuis la collecte de déchets verts en porte à porte à la livraison. Le point critique de contrôle des chargements entrant sur la station fait l'objet d'une renégociation avec les prestataires. Ce changement s'effectue sous la pression forte des inspecteurs de la Direction de l'environnement qui mettent plusieurs fois en demeure le TCO. Des investissements sont réalisés pour vidanger la station, l'étendre et recruter de nouveaux agents. Le compostage des déchets verts, en échec dans les autres intercommunalités de l'île, est inscrit dans les principes directeurs du nouveau syndicat de gestion de déchets. Les projets d'incinération des déchets verts formulés en 2011 sont abandonnés en 2015 au profit d'un renforcement de la filière de compostage.

Un second changement est observé dans la construction des politiques publiques encadrant les activités économiques liées au recyclage des déchets organiques. En 2014, la rédaction de la nouvelle version du Plan de développement rural de La Réunion intègre deux mesures agri-environnementales qui prévoient de subventionner pour les sept ans à venir la construction de stations de recyclage de déchets organiques et l'utilisation de fertilisants organiques normés. Ces deux mesures s'appuient en partie sur des données chiffrées produites au cours du projet. Les agents chargés de leur rédaction étaient déjà favorables au principe de recyclage mais ont été rassurés quant à la crédibilité technique de sa mise en œuvre et ont bénéficié d'un appui politique de la part des différents acteurs institutionnels en charge du dossier. Peu de 
temps après la fin du projet, un comité stratégique «fertilisation organique» est créé au niveau du pôle de compétitivité Qualitropic et un nouvel appel à manifestation d'intérêt est directement inspiré des conclusions du projet. En 2015, le Conseil général maintient le recyclage des déchets verts dans son plan stratégique de gestion des déchets non dangereux. $\mathrm{Au}$ niveau du Conseil régional, le recyclage des matières organiques est inscrit dans le plan stratégique pour l'innovation S3 (Smart Specialization Strategy). Ces trois cas documentés de changements ne proposent pas une application directe des solutions techniques du projet Girovar. Les produits $\mathrm{du}$ projet Girovar sont traduits, adaptés et mobilisés opportunément au sein de différents processus administratifs parallèles d'élaboration d'instruments de politique publique, comme cela a été décrit par Lascoumes et Le Galès (2005) dans des cas similaires. Ce travail de traduction mobilise divers intermédiaires, dont les bureaux d'étude et les agents des services déconcentrés de l'État.

Un troisième changement est observé au niveau de la communauté scientifique et des services techniques d'appui à l'agriculture. Appuyées par le Centre de coopération internationale en recherche agronomique pour le développement (Cirad), les organisations professionnelles (Chambre d'agriculture et eRcane, le centre de recherche sur la canne) mettent en place des essais agronomiques de fertilisation organique afin d'obtenir les références techniques manquantes. Ces essais s'insèrent dans un programme de recherche plus large d'études agronomiques sur la canne à sucre. La mise en évidence de l'absence de données de références et d'un enjeu de société important amènent des chercheurs n'ayant suivi que de loin le projet à insérer des fertilisants organiques dans leur protocole expérimental.

Enfin, une action collective de lobbying est initiée en marge du projet Girovar dès 2012. Elle vise à faire reconnaître par le législateur la spécificité des matières organiques produites à La Réunion. La forte concentration de chrome et de nickel dans les sols d'origine volcanique pollue les composts, litières et autres amendements produits à La Réunion. Les concentrations de ces produits dépassent régulièrement les seuils maximum liés à la norme NF 44051 correspondant aux amendements organiques, ce qui devrait interdire toute commercialisation. Cette action de lobbying rassemble la plupart des partenaires du projet Girovar, d'autres communautés de communes et des entreprises agroalimentaires importantes. Cette action est l'initiative d'un petit collectif de chargés de mission, de responsables techniques et d'ingénieurs qui, dans le cadre du projet Girovar, travaillent ensemble, échangent leurs points de vue, apprennent à se connaître, se découvrent un intérêt commun. Ils initient cette action collective en mobilisant leurs supérieurs hiérarchiques à l'occasion d'une enquête publique visant à réviser cette norme. En 2015, cet épineux dossier est toujours à l'étude.

\section{Une forme de coordination via une reformulation commune du problème}

Sur la base de ces résultats empiriques, nous avons cherché à mettre en évidence la nature de ces relations entre les produits de la concertation et les changements observés. Ces résultats sont synthétisés dans le tableau 1. Par rapport aux catégories proposées par Mazeaud et al. (2012), les produits recensés n'incluent pas de contribution directe sur le contenu de politiques publiques, ce qui n'est pas surprenant pour un projet de recherche, mais des produits «indirects» au niveau des idées et des controverses et via l'apprentissage des acteurs.

À un niveau individuel, l'acquisition de connaissances formelles et informelles sur l'objet de la concertation - les matières organiques et leur recyclage-, sur les autres parties prenantes et sur leurs interdépendances (Daré et al., 2010) contribue à tous les changements recensés. Cet effet est important mais sa portée est limitée : ceux qui apprennent sont pour l'essentiel ceux qui :

- participent aux ateliers de concertation;

- possèdent déjà une certaine connaissance du sujet;

- ont l'opportunité de mettre en pratique ces connaissances dans le cadre de leurs activités professionnelles en parallèle des ateliers.

Les apprentissages réalisés ne sont pas pour eux des découvertes complètes mais une réduction des incertitudes sur le sujet. La contribution s'effectue non pas lors de la concertation mais au niveau des routines, pratiques et activités du quotidien : la manière de contrôler les déchets, la réponse opportune à la commande d'un supérieur hiérarchique, le choix d'un fertilisant plutôt que d'un autre lors d'une campagne agricole.

Deux autres formes d'apprentissage sont observées au niveau collectif: un apprentissage organisationnel (Argyris et Schon, 1996) au sein des organisations formelles, et un apprentissage social (Bouwen et Taillieu, 2004; Reed et al., 2010) au niveau des communautés d'agriculteurs et des cadres techniques. Ces effets dépendent de la nature des différents systèmes institutionnels dans lesquels la concertation est encastrée et des trajectoires déjà existantes. Au niveau de la communauté d'agglomération TCO, le processus de concertation influence l'organisation du travail, le recrutement de nouveaux agents et les orientations stratégiques. Au niveau du territoire, il renforce une communauté existante de fonctionnaires, dont les activités sont liées au problème commun de gestion des matières organiques.

Le dernier produit de la concertation qui contribue aux changements présente une portée plus large. Il s'agit d'une reformulation commune du problème posé et des solutions permettant de le résoudre. Le processus de concertation a permis de traduire les intérêts des acteurs, les contraintes biophysiques, les opportunités technologiques et les pratiques existantes en une proposition à la fois cohérente politiquement, validée scientifiquement et jugée pertinente par des acteurs locaux. Cette reproblématisation commune, que Holm (1995) qualifie de mythe rationalisé (ou mythe institutionnalisé), est un discours combinant un problème à résoudre - ici lutter contre le gaspillage des nutriments - et une solution technique susceptible de résoudre ce problème. Ce discours est institutionnalisé, en ce sens qu'il n'est progressivement plus remis en question. Il est rationalisé en ce sens que les controverses qu'il suscite ont été progressivement traitées au cours du processus de concertation. Cette nouvelle problématisation est ainsi mobilisée par les participants aux ateliers dans leur contexte professionnel pour justifier des interventions, enrôler d'autres collègues et leurs supérieurs hiérarchiques. Elle influence de ce fait des décisions pratiques et 
Table 1. Contributions de la concertation aux changements identifiés.

Table 1. Dialogue outputs contributions to outcomes.

\begin{tabular}{|c|c|c|c|c|}
\hline Changement observé & $\begin{array}{l}\text { Produits de type } \\
\text { «production de } \\
\text { connaissances formelles » } \\
\text { et «apprentissages au } \\
\text { niveau individuel } \\
\text { (technique)» }\end{array}$ & $\begin{array}{l}\text { Produits de type } \\
\text { «apprentissages au niveau } \\
\text { du collectif } \\
\text { (organisationnel ou } \\
\text { social)» }\end{array}$ & Mythe rationalisé & $\begin{array}{l}\text { Contribution d'évènements } \\
\text { externes au changement }\end{array}$ \\
\hline $\begin{array}{l}\text { Amélioration de la } \\
\text { démarche qualité des } \\
\text { déchets verts }\end{array}$ & $\begin{array}{l}\text { Meilleure connaissance } \\
\text { des aspects } \\
\text { environnementaux, } \\
\text { économiques et } \\
\text { agronomiques du } \\
\text { compostage } \\
\text { Meilleure connaissance } \\
\text { des marchés et du produit } \\
\text { vendu }\end{array}$ & $\begin{array}{l}\text { Renforcement important } \\
\text { des compétences du TCO } \\
\text { et de son prestataire en } \\
\text { gestion des déchets et en } \\
\text { gestion commerciale }\end{array}$ & $\begin{array}{l}\text { Le compost ne s'écoule } \\
\text { pas car les agriculteurs le } \\
\text { considèrent comme un } \\
\text { déchet } \\
\text { L'amélioration de la } \\
\text { qualité du compost } \\
\text { permettra de l'écouler }\end{array}$ & $\begin{array}{l}\text { Mises en demeure de la } \\
\text { Direction de } \\
\text { l'environnement, de } \\
\text { l'aménagement et du } \\
\text { logement de la Réunion } \\
\text { (DEAL) } \\
\text { Recrutement d'agents } \\
\text { supplémentaires }\end{array}$ \\
\hline Essais agronomiques & $\begin{array}{l}\text { Meilleure connaissance de } \\
\text { l'état de l'art en matière } \\
\text { de fertilisation organique. } \\
\text { Réévaluation de la } \\
\text { pertinence de la } \\
\text { fertilisation organique }\end{array}$ & $\begin{array}{l}\text { Aucun identifié } \\
\text { explicitement }\end{array}$ & $\begin{array}{l}\text { Les déchets organiques } \\
\text { sont épandus sans } \\
\text { considération de leur } \\
\text { pouvoir fertilisant. } \\
\text { De nouveaux référentiels } \\
\text { agronomiques permettront } \\
\text { aux agriculteurs de } \\
\text { calculer les doses de } \\
\text { fertilisant organique à } \\
\text { épandre }\end{array}$ & $\begin{array}{l}\text { Nouveau programme de } \\
\text { recherches agronomiques } \\
\text { sur canne à sucre }\end{array}$ \\
\hline
\end{tabular}

micro-arrangements au-delà du seul collectif des participants aux ateliers.

La croyance dans ce mythe rationalisé oriente des actions individuelles vers une direction commune sans prise de décision collective. Les agents de la Direction de l'alimentation, de l'agriculture et de la forêt (DAAF) définissent des subventions à l'usage de produits qui n'existent pas encore. Des éleveurs de volaille réservent leur production de litière pour une unité de recyclage encore à concevoir.

\section{Conclusion: Plaidoyer pour une recherche concertée}

La mobilisation d'une double investigation, de part et d'autre de l' «attribution gap», combinée avec une approche ascendante des relations causales a permis de préciser comment les produits de la concertation contribuent à l'évolution de son objet. Dans notre étude de cas, des apprentissages individuels, organisationnels et sociaux et une 
reproblématisation commune que nous qualifions de mythe rationalisé produisent des effets à une certaine distance des arènes de concertation. Ce travail collectif influence des décisions et actions individuelles, au niveau de situations d'action qui relèvent du quotidien des participants aux ateliers, une fois ces derniers de retour chez eux.

Le protocole d'évaluation du projet Girovar proposé dans cet article nécessite de mobiliser des ressources sur toute la durée du projet, à la fois sur les terrains et au sein des arènes de concertation. Une équipe interdisciplinaire est recommandée pour combiner les compétences d'enquête qualitative inductive qui relèvent de sciences humaines et une maîtrise des enjeux techniques liés à l'objet de la concertation.

Les recherches portant sur l'évaluation des processus de concertation mettent l'accent sur les procédures, outils et protocoles permettant d'améliorer les produits directs de ces processus. Pour évaluer leurs contributions à des changements au-delà du seul cercle des participants, il convient d'élargir le niveau d'analyse à un système d'action plus large. La démarche ascendante expérimentée ici, partant des changements pour y retrouver la contribution des produits de la concertation, s'est révélée pertinente pour montrer la complexité des interactions entre les produits du processus de concertation et les dynamiques sociales, institutionnelles et territoriales contingentes.

Cette complexité complique l'analyse des effets de la concertation. Pour autant, chercher à la réduire pour isoler un "principe actif» constitue un biais méthodologique. L'analyse des changements tels qu'ils se produisent met en évidence au contraire l'importance des synergies, des coïncidences, des opportunités saisies entre différentes dynamiques, entre différents systèmes d'action. Il nous semble que c'est précisément parce qu'elle favorise les interactions entre la production de connaissances et le contexte dans lequel ces connaissances peuvent être utiles que la concertation apporte une plus-value à un travail de recherche.

Nos résultats contribuent de ce fait à légitimer le recours à la concertation comme pratique de la recherche. Le projet de recherche «concertée» étudié a contribué à l'évolution du territoire dès ses premières activités via les effets de la concertation. Ces effets ont été favorisés par la diffusion de connaissances et résultats intermédiaires chemin-faisant. En retour, les parties prenantes ont contribué à améliorer la définition des questions et activités de recherche à conduire. Cette modalité de recherche, courante dans des dispositifs de recherche et développement industriels, semble avoir favorisé la contribution de la recherche à l'écologie territoriale.

\section{Références}

Argyris C, Schon D. 1996. Apprentissage organisationnel: théorie, méthode et pratique. Paris: De Boeck.

Barbier R, Larrue C. 2011. Démocratie environnementale et territoires: un bilan d'étape. Participations 1(1): 67-104. DOI: 10.3917/parti.001.0067.

Barret D, Blundo-Canto G, Dabat M-H., Devaux-Spatarakis A, Faure G, Hainzelin E, et al. 2017. IMPRESS (IMPact des REchercheS au Sud). Guide méthodologique ImpresS. Évaluation ex-post des impacts de la recherche agronomique dans les pays du Sud. Montpellier: CIRAD, 96 p. Disponible sur http://agritrop.cirad.fr/586223.
Berkes F. 2009. Evolution of co-management: role of knowledge generation, bridging organizations and social learning. Journal of Environmental Management 90: 1692-1702. DOI: 10.1016/j. jenvman.2008.12.001.

Beuret J, Pennanguer S, Tartarin F. 2006. D'une scène à l'autre, la concertation comme itinéraire. Natures Sciences Sociétés 14: 3042. DOI: $10.1051 / \mathrm{nss}: 2006005$.

Biggs R, Raudsepp-Hearne C, Atkinson-Palombo C, Bohensky E, Boyd E, Cundill G, et al. 2007. Linking futures across scales: a dialog on multiscale scenarios. Ecology and Society 12(1): 17. Available from http://www.ecologyandsociety.org/vol12/iss1/art17/.

Bouwen R, Taillieu T. 2004. Multi-party collaboration as social learning for interdependence: developing relational knowing for sustainable natural resource management. Journal of Community \& Applied Social Psychology 14: 137-153. DOI: 10.1002/casp.777.

CGDD. 2014. Écologie industrielle et territoriale : le guide pour agir dans les territoires. Paris : Collection «RéférenceS » du Commissariat Général au Développement. Disponible sur https://www. ecologique-solidaire.gouv.fr/sites/default/files/EIT\%20-\%201e\% 20guide\%20pour\%20agir\%20dans\%201es\%20territoires.pdf.

Daré W, Van Paassen A, Ducrot R, Mathevet R, Queste J, Trebuil G, et al. 2010. Apprentissage des interdépendances et des dynamiques. In: Étienne M, ed. La modélisation d'accompagnement. Une démarche participative en appui au développement durable. Versailles : QUAE, pp. 223-250.

Douthwaite B, Kuby T, Van De Fliert E, Schulz S. 2003. Impact pathway evaluation: an approach for achieving and attributing impact in complex systems. Agricultural Systems 78: 243-265. DOI: $10.1016 /$ S0308-521X(03)00128-8.

Dufy C, Weber F. 2007. L'ethnographie économique. Paris: La Découverte, $128 \mathrm{p}$.

Étienne M. 2011. La modélisation d'accompagnement, une démarche participative en appui au développement durable. Versailles: QUAE, $367 \mathrm{p}$.

Fazey I, Bunse L, Msika J, Pinke M, Preedy K, Evely A, et al. 2014. Evaluating knowledge exchange in interdisciplinary and multistakeholder research. Global Environmental Change 25: 204-220. DOI: 10.1016/j.gloenvcha.2013.12.012.

Holm P. 1995. The Dynamics of Institutionalization: transformation processes in Norwegian Fisheries. Administrative Science Quarterly 40(3): 398-442. DOI: 10.2307/2393791.

Joly P-B., Colinet L, Gaunand A, Lemarié S, Larédo P, Matt M. 2015. Évaluer l'impact sociétal de la recherche pour apprendre à le gérer: l'approche ASIRPA et l'exemple de la recherche agronomique. Annales des Mines-Gérer et Comprendre 122(4): 31-42. Disponible sur http://www.annales.org/gc/2015/gc122/GC-122-Article-JOLY.pdf.

Lascoumes P, Le Galès P. 2005. Gouverner par les instruments. Paris : Presses de Sciences Po, 370 p.

Lascoumes P, Le Galès P. 2007. Sociologie de l'action publique. Paris: Armand Colin, $128 \mathrm{p}$.

Le Bourhis J.P. 2012. Le gouvernement territorial de l'environnement. In: Barbier R, Boudes P, Bozonnet J-P, Candau J, Dobré M, Lewis N, Rudolf F, eds. Manuel de sociologie de l'environnement. Quebec: Presse de l'Université de Laval, pp. 215-223.

Lynam T, De Jong W, Sheil D, Kusumanto T, Evans K. 2007. A review of tools for incorporating community knowledge, preferences, and values into decision making in natural resources management. Ecology and Society 12(1): 5. Available from http:// www.ecologyandsociety.org/vol12/iss1/art5/.

Mazeaud A, Sa Vilas Boas M-H, Berthomé G-E-K. 2012. Penser les effets de la participation sur l'action publique à partir de ses impensés. Participations 2(1): 53-77. DOI:10.3917/ parti.002.0053. 
Olsson P, Folke C, Berkes F. 2004. Adaptive co-management for building resilience in socio-ecological systems. Environmental Management 34(1): 75-90. DOI: 10.1007/s00267-003-0101-7.

Ostrom E. 1990. Governing the Commons: The evolution of institutions for collective action. Cambridge: Cambridge University Press, 302 p. Queste J. 2016. Concertation et changements : le cas du recyclage des déchets organiques à La Réunion. Thèse de doctorat. Université Grenoble Alpes. https://tel.archives-ouvertes.fr/tel-01433833/.
Reed M, Evely A, Cundill G, Fazey I, Glass J, Laing A, et al. 2010. What is social learning? Ecology and Society 15(4): r1. Available from http://www.ecologyandsociety.org/vol15/iss4/resp1/.

Wassenaar T, Queste J, Paillat J, Saint-macary H. 2015. La coconstruction de filières de recyclage de résidus organiques à $\mathrm{La}$ Réunion. Innovations Agronomiques 43: 161-175. Disponible sur http://www6.inra.fr/ciag/content/download/5605/42543/file/ Vol43-14-Wassenaar.pdf.

Citation de l'article : Queste J, Wassenaar T. 2018. Comment la recherche concertée contribue à l'écologie territoriale. Cah. Agric. 27: 15006 . 